\title{
Answer to the Letter to the Editor of Liang Ding et al. concerning "Comparison of blood loss according to use of aspirin in lumbar fusion patients" (by Park HJ, Kwon KY, Woo JH (2014) Eur Spine J. doi:10.1007/s00586-014-3294-y)
}

\section{Ki-Youn Kwon}

Accepted: 21 June 2014/ Published online: 3 July 2014

(C) Springer-Verlag Berlin Heidelberg 2014

To the editor:

We appreciate the recognition and kind suggestions from the letter.

L. Ding et al. indicated that we did not mention the number of fused levels and the approach of surgery in our 3 groups, respectively.

We agree that we did not mention the number of fused levels and the approach of surgery in Table 1. But, we mentioned this in 'Subjects' as follows: "This study was conducted with patients who had undergone decompression and posterolateral fusion on two segments of the lumbar spine at our institution from September 2008 to January 2011."

I hope that this short explanation will help to better understand our study.

Conflict of interest None.
K.-Y. Kwon ( $\square)$

Department of Orthopedic Surgery, Wonju College of Medicine,

Yonsei University, Ilsan-dong 162, Wonju 220-701,

Republic of Korea

e-mail: techkwon@nate.com 